\title{
Restructurations et gouvernance d'entreprise en Grande-Bretagne : la vente de Rover
}

Restructurings and Corporate Governance in United Kingdom: the sale of Rover

\section{Simon Deakin}

\section{(2) OpenEdition}

12 Journals

Édition électronique

URL : http://journals.openedition.org/travailemploi/3741

DOI : 10.4000/travailemploi.3741

ISSN : 1775-416X

Éditeur

DARES - Ministère du Travail

\section{Édition imprimée}

Date de publication : 15 mars 2007

Pagination : $51-58$

ISBN : ISSN : 0224-4365

ISSN : 0224-4365

\section{Référence électronique}

Simon Deakin, «Restructurations et gouvernance d'entreprise en Grande-Bretagne : la vente de Rover », Travail et Emploi [En ligne], 109 | janvier-mars 2007, mis en ligne le 15 mars 2009, consulté le 19 avril 2019. URL : http://journals.openedition.org/travailemploi/3741 ; DOI : 10.4000/ travailemploi.3741 


\title{
Restructurations et gouvernance d'entreprise en Grande-Bretagne: la vente de Rover
}

\author{
Simon Deakin*
}

\begin{abstract}
Lorsque l'entreprise Rover a été confrontée en 2000 à une annonce d'acquisition par une société de capital-risque, les lois accordant aux travailleurs et à leurs représentants des droits d'information et de consultation ont semblé jouer un rôle important dans le succès d'un acquéreur rival, qui représentait les intérêts des salariés et de la région. D'un point de vue comparatif, l'article met en évidence l'impact des directives communautaires en Grande-Bretagne, rappelant les progrès importants réalisés grâce à la généralisation des droits fondamentaux relatifs à l'information et la consultation des travailleurs dans les pays de l'Union qui n'ont pas construit un droit du travail performant. Si la directive européenne de 2002, qui généralise l'information et la consultation des travailleurs, avec des différences persistantes entre modèle britannique et modèles continentaux, avait été en vigueur en Grande Bretagne en 2002, ces derniers auraient peut-être pu faire entendre leur voix y compris dans la seconde phase de la reprise. L'exemple de Rover montre aussi que, si un partenariat se crée pour offrir une alternative industrielle (et non seulement financière), le point central consiste à construire des droits conférant un pouvoir sur la décision économique aux parties prenantes dans la nouvelle structure sociétaire, et en particulier aux organisations syndicales. Dans les discussions relatives à la corporate governance en Europe, des dispositions reconnaissant des droits de vote aux représentants des salariés en cas de reprise lors des restructurations pourraient représenter une étape considérable vers la mise en place d'alternatives favorables à l'emploi en Europe.
\end{abstract}

Le long déclin de l'industrie automobile britannique a inquiété les spécialistes de l'industrie et les experts des relations professionnelles pendant plusieurs dizaines d'années. À la fin des années 1990, la notion de gouvernance d'entreprise a ouvert une nouvelle perspective( 1 ) puisque c'est dans ce cadre que sont prises les décisions de restructurations, en particulier en cas de cession d'entreprise ou de cession d'actifs.

L'intérêt pour les aspects de gouvernance a été déclenché par des événements liés à la proposition de BMW de vendre le groupe Rover, le dernier constructeur automobile britannique implanté sur le territoire national, à une société de capital-risque, Alchemy Partners, dans les premiers mois de l'année 2000.

\footnotetext{
(*) Centre for Business Research, université de Cambridge. (1) Note de la rédaction : ce thème est bien connu, en particulier en Grande-Bretagne dans ses interférences avec le droit du travail. Sur le terrain des approches de corporate governance, les actionnaires shareholders sont opposés aux parties prenantes de l'entreprise, stakeholders, l'objectif étant grâce à des règles d'organisations des pouvoirs et par un management indépendant de mettre en place des compromis entre toutes les parties prenantes favorisant la performance de l'entreprise (avec une limitant des coûts de transaction). Les salariés comme les organisations syndicales sont des parties prenantes au même titre que les fournisseurs, les banquiers, les clients de l'entreprise, etc. S'opposent sur le terrain de la corporate governance plusieurs théories sur la façon d'organiser l'équilibre entre shareholders and stakeholders, la plus connue étant celle de la shareholdervalue fondée sur l'exigence de rentabilité maximale pour les actionnaires, voir ci après.
}

Alors que la transaction était sur le point d'aboutir, la société Alchemy s'est retirée, laissant la voie libre au «sauvetage» de Rover par le consortium Phœnix. Phœnix a rassemblé plusieurs parties prenantes: des anciens cadres dirigeants de Rover dont un grand nombre avait quitté le groupe lorsqu'il avait été repris par BMW, des salariés et leurs représentants, parmi lesquels de nombreux syndicats, des fournisseurs et des clients, y compris un large réseau de concessionnaires automobiles, et des administrations locales de la région des West Midlands en Angleterre, dans laquelle la plupart des usines Rover étaient implantées. En raison de cette implication des parties prenantes, les aspects de gouvernance de la vente de Rover à Phœenix ont fait l'objet d'une attention considérable.

La vente de Rover a permis d'étudier l'influence des mécanismes d'information et de consultation des salariés (qui sont courants en Europe continentale, mais sont rarement employés dans le contexte britannique) sur le résultat d'une transaction commerciale, puisqu'ils ont apparemment joué un rôle dans ce cas de figure.

Depuis le sauvetage de Rover, cette forme insolite de gouvernance d'entreprise continue de susciter l'intérêt, voire même la controverse, en suggérant que les structures d'entreprise complexes diluent la responsabilité de leurs dirigeants. Le sort de Rover s'est trouvé lié à des débats plus vastes sur les mérites de deux modèles contradictoires de gouvernance 
d'entreprise: le premier repose sur une pratique britannique traditionnelle, qui donne la priorité aux intérêts financiers et en particulier à la norme de la valeur actionnariale, tandis que le second privilégie une logique de coopération entre des groupes de parties prenantes incluant les salariés, les fournisseurs et les régions concernées.

L'insolvabilité de la société en 2005 constitue le moment idéal pour évaluer ce débat(2).

\section{L’arrière-plan juridique}

Conformément au droit du travail au RoyaumeUni, le processus de prise de décision des dirigeants confrontés à une restructuration d'entreprise est influencé principalement par la législation accordant des droits d'information et de consultation aux représentants des salariés et par les dispositions de la législation sur la protection des salariés qui régissent la responsabilité potentielle des employeurs dans le cas de congédiement abusif ou de licenciement économique. Grâce à l'adoption des directives européennes relatives aux comités d'entreprise européens (3) et à l'information et la consultation des salariés (4) et leur transposition dans le droit britannique via les réglementations TICER et ICER, (5) de nombreuses entreprises britanniques, à partir d'une certaine taille, sont soumises à l'obligation de mettre en place des mécanismes de dialogue social pour les représentants du personnel, sur des questions incluant les fusions et acquisitions.

Les réglementations ICER, en particulier, pourraient avoir un impact significatif sur les pratiques de consultation des salariés au Royaume-Uni en fournissant un cadre juridique pour le développement d'une «deuxième voie» de représentation des travailleurs, quelque peu similaire aux modèles de participation au niveau de l'entreprise qui existent en Europe continentale.

Cependant, comme ces réglementations sont appliquées depuis peu (6 avril 2005), leurs effets potentiels s'inscriront dans le futur. Pour le cas

(2) Cet article a pour but d'étudier le rôle des lois relatives à la consultation des salariés et la structure de la propriété et du contrôle de Rover à la suite de son acquisition par Phœnix et non les modalités du plan social mis en œuvre à la suite de l'insolvabilité de Rover en 2005, à ce sujet, consulter LEFRESNE, 2005.

(3) Directive 94/45/CE du 22 septembre 1994 portant sur l'institution d'un comité d'entreprise européen ou d'une procédure dans les entreprises et les groupes de dimension communautaire en vue d'informer et de consulter les employés, qui été étendue au Royaume-Uni en 1997 à la suite de l'expiration de la dérogation à la politique sociale, définie par l'accord de Maastricht, qui avait été accordée au Royaume-Uni.

(4) Directive 2002/14/CE du 11 mars 2002 établissant un cadre général relatif à l'information et la consultation des travailleurs dans la Communauté européenne.

(5) Réglementations transnationales afférentes à l'information et la consultation transnationale des employés («TICER»), SI 1999/3323, et réglementations afférentes à l'information et la consultation des employés («ICER»), SI 2004/3426.
Rover, les règles TUPE, établies de longue date, ont été effectives: elles prévoient la consultation des salariés dans le cas de licenciements collectifs (6) et de transferts d'entreprise(7).

Pour Armour et Deakin (2003), ces lois sur l'information et la consultation fournissent aux travailleurs des droits à la parole qui respectent les intérêts des salariés participant depuis longtemps à la vie de l'entreprise. À ce titre, ils ont la capacité de déplacer l'équilibre des forces en présence dans la gouvernance de l'entreprise et de modifier le résultat des restructurations. Leur démonstration s'appuie sur trois propositions qui reposent sur les concepts de droits à la parole «contingents», de revendications de nature patrimoniale, et de représentation monopolistique:

1) La législation sur la protection de l'emploi fournit des droits à la parole «contingents» aux salariés, c'est-à-dire des droits qui sont déclenchés par une altération significative de la forme de l'entreprise constituant une menace pour le «capital humain spécifique à cette entreprise», comme c'est le cas pour les licenciements collectifs ou la vente de l'entreprise, en totalité ou en partie, à un tiers.

Selon la législation du travail applicable au Royaume-Uni, les salariés faisant l'objet d'un licenciement économique ont le droit statutaire de recevoir une indemnisation(8); ils peuvent également obtenir une indemnisation en cas de congédiement abusif si les licenciements ont été opérés sur la base d'une sélection inéquitable (la réintégration, bien qu'elle soit possible en principe, se produit rarement dans ce contexte).(9) Ces dispositions ne concernent pas la question de la gouvernance. En outre, lorsqu'un employeur envisage des licenciements massifs ou est sur le point de finaliser une transaction commerciale entraînant un transfert d'entreprise (selon la définition retenue par les réglementations TUPE), il doit consulter les représentants du personnel afin de conclure un accord sur les moyens de protéger les emplois et les «droits acquis» des salariés $(10)$. Un défaut de consultation peut se traduire par une obli-

(6) Loi «TULRCA» de 1992 (Trade Union and Labour Relations (Consolidation) Act), art. 188 et suivants, dérivée à l'origine de la directive 75/129/CE sur le rapprochement des législations des États membres relatives aux licenciements collectifs, codifiée ultérieurement par la directive 1998/59/CE. (7) Réglementations TUPE (Transfer of Undertakings (Protection of Employment), SI 1981/1794 (TUPE), regs 10$11 \mathrm{~A}$, dérivées à l'origine de la directive $77 / 187 / \mathrm{CE}$ sur le rapprochement des législations des États membres relatives au maintien des droits des travailleurs dans le cas de transferts d'entreprises, d'établissements ou de parties d'établissements ou d'entreprises, codifiée ultérieurement dans la directive 2001/23/CE. Les réglementations de 1981 ont été remplacées par une nouvelle version codifiée des TUPE, le 6 avril 2006, pour y incorporer un certain nombre d'amendements.

(8) Loi britannique de 1996 sur les droits afférents à l'emploi, partie XI.

(9) Loi britannique de 1996 sur les droits afférents à l'emploi, partie X.

(10) Transposition de la directive communautaire. 
gation de versement d'indemnités civiles par l'employeur, laquelle contient une sanction significative: l'employeur doit payer une somme équivalente aux salaires de tous les travailleurs concernés par cette violation de la loi pendant toute la durée de la période qui aurait dû être consacrée à la consultation (jusqu'à une limite statutaire basée sur des salaires hebdomadaires «normaux») en plus de leurs droits contractuels existants.

2) Ces revendications s'apparentent à un droit patrimonial, qui apparaît lorsque des droits existants ou «acquis» des salariés engagent automatiquement des parties tierces, telles que l'acquéreur ou le cessionnaire de l'entreprise.

Le non-respect par un employeur des droits d'information et de consultation de ses employés n'a pas d'effet sur la validité de la transaction commerciale (la vente ou la délocalisation de l'entreprise, par exemple) ou sur son impact sur la relation de travail. Les tribunaux anglais ont refusé d'admettre qu'un congédiement abusif dans le cadre d'un transfert d'entreprise soit nul et non avenu(11). Cependant, la sanction de l'indemnité civile, aux termes de la législation britannique, peut avoir un effet dissuasif puissant. En doublant concrètement (jusqu'à une certaine limite) les salaires des travailleurs pendant plusieurs semaines, voire des mois, la somme due par l'employeur devient vite très élevée et grignote les revenus et actifs dont pourraient autrement disposer les actionnaires et, dans certains cas, les créanciers. Par voie de conséquence, cela peut conduire à une remise en question d'une stratégie managériale spécifique en matière de restructuration.

Les réglementations TUPE vont encore plus loin en garantissant que les revendications des salariés ne puissent pas être annulées par un simple transfert de la propriété des autres actifs de l'entreprise. En effet, elles contiennent un mécanisme de novation des contrats de travail dans le cas d'un transfert de l'entreprise, en totalité ou en partie. Les «droits acquis» du salarié, à la fois contractuels et statutaires, sont automatiquement reportés dans la relation de travail avec le nouvel employeur. Cela ne s'applique pas uniquement aux conditions d'engagement et aux droits statutaires en matière d'emploi. L'employeur cessionnaire hérite également des revendications liées à l'emploi qui avaient été formulées à l'encontre du cédant, y compris toute responsabilité pour manquement à la législation relative à l'information et la consultation et toute demande d'indemnisation non encore réglée pour licenciement économique et congédiement abusif.

3) La législation relative à l'information et la consultation a pour effet de collectiviser les intérêts divergents des salariés de manière à éviter les coûts

(11) Wilson contre St. Helens BC/Baxendale contre British Fuels [1998] IRLR 706, même situation en droit français. considérables engendrés par la renégociation des conditions des contrats de travail, en conférant ces droits de négociation à des mandataires désignés qui détiennent, à cette fin, des droits de représentation monopolistique.

La législation sur l'insolvabilité, en raison de la manière dont elle collectivise les droits des créanciers, fournit un mécanisme de résolution des divergences d'intérêts entre les créanciers et les autres parties prenantes impliquées dans le processus de sauvetage d'une entreprise (voir HART MOORE, 1997, repris par Armour, DeAkin, 2001). De même, il est possible de résoudre les divergences d'intérêts entre les différents salariés, tout du moins en partie, en faisant intervenir les représentants des travailleurs auxquels sont accordés des droits de représentation monopolistique dans le cadre de la législation relative à l'information et la consultation. Du point de vue de la performance, un tel mécanisme est important car il permet de réduire les coûts de transaction associés aux formes de gouvernance d'entreprise impliquant des parties prenantes multiples.

Conformément à la législation en vigueur au Royaume-Uni, selon laquelle un syndicat indépendant est reconnu par l'employeur comme interlocuteur dans les négociations collectives sur les termes et conditions applicables aux salariés concernés, l'employeur doit consulter ledit syndicat sur les questions relatives aux salariés concernés par les licenciements économiques ou un transfert d'entreprise, à l'exclusion de tout autre organisme. Lorsqu'il n'existe pas de syndicat reconnu, l'employeur doit consulter les représentants du personnel qui ont été élus ou désignés selon les procédures définies par la législation.

La question reste ouverte quant à l'étendue de la dérogation aux droits statutaires qu'autorise la loi, et donc à l'espace de négociation. Les rédacteurs de la directive européenne de 1977 avaient clairement l'intention de laisser un espace de négociation sur les droits acquis des employés. La directive contenait une disposition permettant à un État membre d'autoriser des variations de termes et de conditions dans les conventions collectives applicables aux employés concernés par le transfert d'entreprise, un an après le transfert effectif (à moins que le cessionnaire ait été lié d'une autre manière par les termes de la convention collective en vigueur au moment du transfert)(12). Cependant, une série d'amendements récents à cette directive stipulent spécifiquement qu'une négociation entre l'employeur et les représentants du personnel doit avoir lieu en cas d'insolvabilité (13).

(12) Directive 77/187/CE, art. 3 (3) (il s'agissait de l'art. 3 (2) dans la directive d'origine, avant les amendements apportés par la directive $98 / 50 / \mathrm{CE}$.

(13) Directive 2001/23/CE, art 5 (2) (b). Voir Deakin, Morris, 2005: 560-1 sur les propositions de transposition de cette disposition au Royaume-Uni, qui est entrée en vigueur le 6 avril 2006. 
Le cas Rover: avant et après le sauvetage

Un certain nombre de restructurations d'entreprises impliquant une insolvabilité et des transferts d'entreprise ont déjà fait l'objet de recherches (Armour, Deakin, 2000, 2003, 2004) mais le cas le plus important reste la vente du constructeur automobile Rover par BMW au consortium Phœnix en 2000. La vente de Rover permet d'étudier l'influence des mécanismes d'information et de consultation des salariés (qui sont courants en Europe continentale, mais rarement employés dans le contexte britannique car non obligatoires avant 2005, sauf dans des cas de licenciements collectifs ou transferts d'entreprise) sur le résultat d'une transaction commerciale, puisqu'ils ont apparemment joué un rôle dans ce cas de figure.

Les négociations pour la vente de RoveràAlchemy Partners, une société de capital-risque implantée à Londres, commencent en coulisses en octobre 1999 et sont rendues publiques lorsque BMW annonce son intention de vendre Rover et les marques $M G$ à Alchemy ainsi que son usine de Longbridge. BMW déclare également qu'il a l'intention de conserver l'usine de Cowley pour la production de la nouvelle Mini et annonce peu de temps après qu'il vendrait à Ford la division rentable qu'est Land Rover. Il devient vite évident qu'Alchemy a l'intention de réduire significativement l'échelle de production à Longbridge, en se concentrant sur la petite niche de marché que représentent les modèles $\mathrm{MG}$, au détriment des marques Rover. De ce fait, son offre est reçue avec hostilité tant par les syndicats que par les autorités locales de la région des West Midlands. Fin mars, une offre rivale, qui deviendra le consortium Phœenix, est annoncée. Celle-ci est présentée en temps utile par John Towers, un ancien dirigeant de Rover, et porte sur la conservation de Longbridge comme site de production automobile de masse. Cependant, pendant la plus grande partie du mois d'avril, BMW refuse de négocier avec Phœnix et continue d'agir en vue de finaliser la vente de Rover à Alchemy.

Alors que la vente à Alchemy est imminente, six nouveaux domaines d'activité stratégiques sont créés sous forme de filiales du groupe Rover. Les actions de ces filiales sont alors achetées par la holding britannique de BMW. Cette opération visait à établir une séparation entre les parties du groupe qui resteraient chez BMW (notamment, le site de fabrication de moteurs et l'usine de Cowley qui devait être conservée pour produire la nouvelle Mini pour BMW), les parties qui seraient vendues à Ford (Land Rover) et les parties qui resteraient à Alchemy. Sept filiales ont donc été créées en tout, si l'on inclut le groupe Rover, c'est-à-dire la société d'origine qui a donné naissance aux six autres. L'intention était de concrétiser la vente des actifs de Longbridge à Alchemy sous forme d'une cession d'actions de la partie résiduelle du Groupe Rover.

BMW a structuré sa proposition de vente de Rover à Alchemy de manière à pouvoir soutenir qu'il s'agissait d'une cession d'actions - et non d'un changement formel d'employeur-ce qui rendait la législation TUPE inapplicable. Dans l'hypothèse d'une cession d'actions, les salariés conservaient leurs droits statutaires et contractuels acquis au moment de la reprise par Alchemy, mais aucun employeur n'était tenu de consulter ou d'informer les représentants du personnel. De même, Alchemy n'était pas rigoureusement tenu de préserver les termes et conditions des contrats après la cession.

Toutefois, on pouvait aussi soutenir que les cessions avaient eu lieu dans le cadre de la création de six nouvelles filiales auxquelles avaient été affectés les salariés des domaines d'activité stratégiques concernés. En effet, cette opération n'était pas éloignée d'une procédure de «cantonnement» qui, selon la jurisprudence, pouvait être considérée comme une transaction unique au cours de laquelle la holding britannique de BMW se substituait à Rover dans le rôle de l'employeur. La réticence des tribunaux à accepter le contournement de la législation TUPE a été réaffirmée dans une décision de la Haute Cour dans le cadre du jugement In the Matter of Maxwell Fleet and Facilities Management Ltd. à l'époque de la proposition de vente(14). Les avocats représentant un groupe de cadres dirigeants ont avancé l'argument que la création de la société Rover «résiduelle», en anticipation de la vente d'actions à Alchemy, était similaire à une procédure de cantonnement, et en tant que telle, tomberait en temps opportun sous le coup de la législation TUPE. Ce dernier point en faveur des salariés était moins clairement établi, mais le doute était permis.

Ni le Groupe Rover, ni l'une ou l'autre filiale britannique de BMW n'avaient entamé de procédure de consultation des représentants des travailleurs sur les transferts d'entreprise. Les représentants concernés étaient les syndicats reconnus qui avaient donc l'occasion d'intenter une action en justice directement. Les salariés ont également argué du fait qu'étant donné que BMW et Alchemy avaient tous deux indiqué que des licenciements résulteraient de la vente du groupe Rover et des autres filiales, les lois afférentes à la consultation en cas de licenciement n'avaient pas été respectées. Dans le jugement Kerry Foods Ltd. contre Creber(15), l'EAT (tribunal d'appel britannique en matière d'emploi) avait statué, quelques mois auparavant, que la responsabilité d'un employeur, dans le contexte des indemnités civiles, pouvait être transférée, en vertu de l'article 5 de la législation TUPE au nouvel employeur (allant ainsi à l'encontre de la jurisprudence antérieure). Cela signifiait que tout

(14) [2000] IRLR 368, 372-373.

(15) [2000] IRLR 10. 
manquement par BMW ou ses filiales aux obligations d'information et de consultation serait transféré aux acquéreurs des domaines d'activités. La Cour européenne de justice dans l'affaire Allen v. Amalgamated Construction Co. Ltd.(16) a confirmé qu'il n'y avait aucune raison que les transferts d'entreprises entre sociétés d'un même groupe ne puissent pas être régis par la directive sur les droits acquis, renforçant ainsi les revendications des salariés. Sur ces différentes bases, les syndicats ont déposé des demandes d'indemnités civiles, applicables aux 28000 employés de l'ancien Groupe Rover, auprès des conseils des prud'hommes (employment tribunals) à la fin du mois d'avril. Par ailleurs, des mesures ont été prises pour préparer des actions individuelles pour rupture de contrat dans le cadre des congédiements effectués en contravention des accords de «licenciement non obligatoire» conclus entre Rover et les syndicats, considérés comme intégrés aux contrats de travail des salariés. Globalement, la valeur potentielle de ces actions dépassait 300 millions de livres sterling.

C'est dans ce contexte qu'Alchemy a rompu les négociations avec BMW le 27 avril, soit environ un jour avant la date de finalisation prévue. On ne sait pas clairement ce qui a déclenché la rupture de la transaction avec Alchemy. Des rapports rédigés après coup suggèrent qu'Alchemy a été dissuadé de conclure la transaction face à la perspective de reprise des engagements des plans de retraite et des créances du réseau de concessionnaires Rover dont le volume restait flou(17).

$\mathrm{Au}$ moment de l'échec des négociations, des articles dans la presse ont suggéré que le déclencheur avait été l'insistance de BMW à demander qu'Alchemy lui verse une indemnité pour des actions éventuelles portant (entre autres) sur le non-respect de la législation sur 1'information et la consultation et des créances contractuelles pour congédiement abusif. Il a été rapporté qu'Alchemy était prêt à payer 50 millions de livres sterling pour la société si BMW acceptait de payer certaines dettes découlant de la restructuration, mais ne voulait pas consentir à verser une indemnité pouvant atteindre des «centaines et centaines de millions de livres» (18).

La vente au consortium Phœnix a été finalisée le 10 mai. Le groupe Rover a été vendu pour une valeur nominale de 10 livres, mais BMW a payé 575 millions de livres pour participer à certains frais de fonctionnement. La direction du consortium Phœnix a incité les syndicats à accepter de renoncer à toute action portant sur le non-respect de BMW (via Rover) de la procédure de consultation au moment de l'annonce de la vente potentielle à Alchemy. Bien que les syndicats n'aient pas accepté

(16) [2000] IRLR 119

(17) BRADY, LORENZ, $2002: 186-8$.

(18) Financial Times, «How the Alchemy deal fell apart», $1^{\text {er }}$ mai 2000 . de renoncer à toute poursuite, un accord en ce sens a été conclu en se basant sur le fait que la consultation entre Phœnix et les syndicats avait été entamée à une date antérieure. Cela permettait d'économiser 100 millions de livres. En retour, Phœnix acceptait d'insérer des conditions de licenciement plus favorables dans les contrats de travail des employés de Longbridge. Il a été possible d'économiser 200 autres millions de livres lorsque Phœnix a décidé de licencier moins de 1000 travailleurs à Longbridge, évitant ainsi des demandes d'indemnités de licenciement à large échelle.

Les avocats représentant les syndicats ont fait à l'époque le commentaire suivant: «Les syndicats avaient l'intention de mettre le plus d'obstacles [juridiques] possibles sur le chemin de la société Alchemy, sans pour autant la faire tomber... et donner un chèque en blanc à John Towers là la tête du consortium Phœnix]»(19). Selon les avocats représentant la direction de Rover, «les mêmes questions relatives à 1'emploi qui avaient diabolisé l'offre Alchemy ont été de nouveau posées mais ont été résolues par la discussion, la négociation et un accord $\gg(20)$. Sans renoncer formellement à leurs revendications, les salariés et leurs représentants se sont trouvés dans une position où leur implication dans le processus de sauvetage avait eu pour effet de faire accepter l'offre qui entendait conserver Rover comme un producteur automobile de masse.

Comme l'a déclaré un des avocats des syndicats, «nous avons lancé plein de choses en l'air dans 1'espoir que cela [l'offre] aille mal pour Alchemy» (21). Par exemple, nous n'avons jamais approfondi la question de savoir si un transfert TUPE se serait produit si Alchemy avait acheté des actions dans la société résiduelle Rover; nous ne savions pas clairement dans quelle mesure on pouvait lever le voile recouvrant l'entreprise, si tant est que cela eut été possible, pour rattacher les diverses dettes contractées par les filiales de BMW à sa holding britannique. Par ailleurs, la question du statut contractuel des clauses d'origine concernant le licenciement non obligatoire est restée sans réponse. Si ces questions avaient été posées au tribunal, leurs réponses auraient aussi bien pu être en faveur de l'employeur. Toutefois, pendant ces négociations difficiles et complexes, ces questions juridiques posaient des risques potentiels énormes pour n'importe quel acquéreur de Rover ayant l'intention de procéder à des restructurations à large échelle. À cet égard, Phœnix présentait l'avantage de vouloir procéder à une restructuration limitée.

La structure de gouvernance mise en place après la vente de la société, qui était devenue indépendante

(19) Financial Times, «Claims that hurt Alchemy bid to be waived », 9 mai 2000 .

(20) Ibidem.

(21) Ibidem. 
et s'appelait MG Rover, devait refléter les intérêts des divers groupes de parties prenantes qui avaient participé au sauvetage par Phœnix, notamment les salariés et les fournisseurs et concessionnaires locaux qui comptaient sur Rover pour le maintien de leurs activités. La nouvelle équipe managériale avait décidé de réserver $35 \%$ des actions pour les salariés et $25 \%$ pour les concessionnaires. Les actions des concessionnaires étaient détenues par l'intermédiaire d'une société de fiducie tandis que les titres détenus par des concessionnaires individuels faisaient l'objet d'une revalorisation périodique selon une formule d'échelle mobile se basant sur les ventes annuelles des véhicules Rover. Cette décision de répartition de la propriété sous cette forme a été bien accueillie, du moins dans un premier temps et a contribué à renforcer l'idée que la société serait gérée selon les orientations définies par les parties prenantes(22). Néanmoins, la structure mise en place par les nouveaux dirigeants de Rover a vite soulevé de délicates questions.

Le problème central était la structure d'entreprise mise en place par la nouvelle équipe pour gérer la «dot» transmise à Rover par BMW. Au cours de la transaction visant à se débarrasser de Rover, BMW avait consenti à mettre plus de 500 millions de livres à la disposition de la nouvelle société, essentiellement sous forme de prêt, mais à des conditions tellement généreuses qu'il était peu probable qu'il soit jamais remboursé en totalité : en effet, le prêt devrait être remboursé si Rover était revendu dans les trois ans suivant la vente (ce qui ne s'est pas produit) et l'échelonnement du remboursement ne commençait qu'au moment où Rover ferait à nouveau des bénéfices (ce qui n'était jamais le cas). Dans tous les cas, il n'était pas remboursable pendant cinquante ans (23). La nouvelle équipe managériale de Rover avait opté initialement pour un versement de la «dot» via une entité morale qu'elle avait créée pour gérer la transaction Rover, à savoir la société Techtronic. Une nouvelle holding, MG Rover Holdings, est créée en 2000 pour être renommée Phœnix Venture Holdings (PVH) en 2002.

PVH devient la société de portefeuille pour MG Rover et un certain nombre d'autres filiales. Les groupes de parties prenantes, c'est-à-dire les salariés et les concessionnaires, conservent leurs titres participatifs au sein de Rover sous forme de titres PVH sans droit de vote; les quatre fondateurs de Phœnix, menés par John Towers, détiennent entre eux 40\% des actions et $100 \%$ des titres PVH comportant un droit de vote. Selon les termes des statuts constitutifs de PVH, les groupes de «parties prenantes» avaient seulement le droit de recevoir un dividende sur les revenus versés par à $\mathrm{PVH}$ par MG Rover, c'est-à-dire la branche de construction automobile du groupe.

(22) Brady, LoREnZ, 2002: 213 et 221-3.

(23) Brady, Lorenz, 2002: 209.
MG Rover n'a jamais fait de bénéfice; en 2004 son bilan présentait un passif de plus de 200 millions de livres par rapport à son actif. Toutefois, d'autres parties du groupe PVH étaient rentables, en grande partie en raison des ventes immobilières réalisées par $\mathrm{PVH}$, notamment la vente et la cession-bail du site principal de Longbridge (24). Bien que les nouveaux dirigeants de Rover aient défendu cette structure en arguant qu'il s'agissait d'un mécanisme d'injection d'investissements dans l'activité-mère de construction automobile, d'autres l'ont décrit comme un dispositif de protection de leurs intérêts contre une faillite possible de MG Rover: «Si Rover devait fermer, environ 5000 salariés de son usine de Longbridge à Birmingham seraient sans emploi, la Grande-Bretagne perdrait son dernier constructeur automobile de masse indépendant et les quatre fondateurs de Phœnix seraient aux commandes d'une société survivante valant environ 70 millions de livres»(25).

Cette inquiétude s'est étendue aux syndicats qui avaient été le plus impliqués dans le sauvetage et la réhabilitation de la société. L'équipe négociatrice des syndicats était dirigée à l'époque par Tony Woodley, qui était alors un responsable du syndicat TGWU (Transport and General Workers'Union) au niveau national et avait élu secrétaire général du TGWU en 2003. En mars 2004, Tony Woodley fait publier une lettre ouverte adressée aux dirigeants de Rover, qui demande l'intégration des différentes sociétés du groupe Rover dans une seule structure d'entreprise et la nomination de directeurs indépendants au conseil de direction du groupe. D'après les termes de sa lettre, la réintégration était une nécessité pour "faire comprendre très clairement que personne n'avait rien à gagner de la disparition de la construction automobile et personne ne pouvait envisager une éventualité aussi désastreuse »(26).

À l'origine de ces inquiétudes était l'incapacité constante de l'activité métier, à savoir la fabrication de véhicules, à revenir à une situation bénéficiaire. Un certain nombre d'obstacles importants barraient la route à un maintien sur le long terme. Le premier était l'usine de Longbridge proprement dite. Ce site avait été le premier à être construit dans les années 1930 et avait fait l'objet d'investissements limités depuis le début des années 1980. Selon l'avis des experts de l'industrie automobile, il souffrait d'une capacité excédentaire résultant d'un défaut constant à rationaliser et moderniser son infrastructure (27). La deuxième difficulté avait sa source dans la perception générale que Rover était une «marque

(24) Les informations de ce paragraphes sont reprises de l'article Rover's financial rebuild publié dans le journal The Guardian, le 2 mars 2004

(25) Idem.

(26) Union ally calls for independent Rover directors The Guardian, 10 mars 2004.

(27) ProfesseurKumarBhattacharyaduWarwickManufacturing Group, cité dans BRADY, LoRENZ, 2002: 111-112. 
en voie d'extinction», qui n'était plus associée à la qualité ou à l'innovation(28). Ces deux problèmes ont été exacerbés par la manière dont BMW est sorti de la société: BMW conservait la marque Mini (et a beaucoup contribué à la réhabiliter) ainsi que la chaîne de production relativement performante de Cowley tout en vendant à bon prix à Ford la division rentable qu'était Land Rover. Par conséquent, sur l'ensemble de l'entité achetée par BMW en 1994, la nouvelle société Rover se retrouvait avec les composantes ayant le moins de valeur.

Ces problèmes n'ont fait que croître et embellir jusqu'en 2005 et, après l'échec des pourparlers de fusion avec la société chinoise SAIC (implantée à Shanghai), ce qui ne l'a pas empêché d'acquérir des droits de propriété intellectuelle significatifs, la société Rover a été déclarée insolvable et les salariés restants ont été licenciés (pour les détails, se reporter à LEFRESNE, 2005). Les administrateurs ont organisé une vente du fonds de commerce à une autre société chinoise, Nanjing Automobile, qui avait annoncé son intention de relancer la production à Longbridge, mais ne l'a pas encore fait à ce jour.

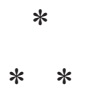

Le sauvetage de Rover est dû en grande partie à l'influence du modèle de consultation des salariés en vigueur en Europe continentale, qui est illustré par la directive européenne sur les droits acquis et appliqué au Royaume-Uni par le biais des réglementations TUPE. Si cette législation n'avait pas existé, il est possible que la vente de Rover à Alchemy aurait eu lieu et que l'offre Phœnix orientée sur les parties prenantes aurait été rejetée.

L'échec ultérieur de Phœnix a néanmoins jeté le doute sur la signification de l'application de la législation TUPE en 2000 dans le but de préserver l'emploi au sein de Rover. Le succès a été de courte durée. Il est possible que la dégradation progressive de l'emploi sous la direction de Phœnix ait été préférable à une perte abrupte de presque 30000 emplois, sans compter les autres pertes d'emploi au niveau des fournisseurs et des concessionnaires, ce qui était une possibilité en 2000. Nous discernons encore mal les leçons plus vastes à tirer de la période postérieure au sauvetage puisque nous attendons la fin d'une enquête du DTI (ministère britannique du Commerce et de l'Industrie) sur la structure et l'organisation du groupe Phœnix. Cependant, une caractéristique frappante de la structure de gouvernance adoptée par Phœnix apparaît déjà clairement : elle n'a fourni aucun mécanisme efficace d'exercice du droit de parole aux salariés: les actions détenues pour le compte des salariés n'étaient pas accompagnées de droit de vote et aucun représentant des travailleurs n'a été désigné pour faire partie de l'équipe de direction de la société (bien qu'un poste de directeur ait apparemment été offert à Tony Woodley, représentant du syndicat TGWU, qui l'a refusé).

Certains attribuent la faillite de Rover à l'intervention inutile de la loi sur la protection de l'emploi en 2000: sans elle, une version dégraissée de la société aurait survécu sous le plan établi par Alchemy. On peut cependant penser que l'essence même du plan d'Alchemy est l'option qui a finalement été adoptée par Phœnix puis par les nouveaux propriétaires chinois à partir de ce qui est resté de Rover. Toutefois, on peut aussi considérer que la vente de la société à Phœnix en 2002 représentait une véritable occasion de bâtir la réussite d'une entreprise sur la base de partenariats innovants.

Cependant, la structure juridique de la représentation des salariés au Royaume-Uni n'a pas été adaptée sur ce terrain. À la différence des modèles de participation des salariés qui sont appliqués en Europe continentale, le droit du travail britannique, à ce moment, disposait que la nature «contingente» des droits à la parole fournis par la loi ne pouvaient s'exercer que sur des périodes de temps limitées, lorsqu'une restructuration était envisagée ou mise en place, et non pas de manière continue. Lorsque la vente à Phœnix a été finalisée, l'occasion pour les salariés de faire entendre leur voix collective sur les questions de gouvernance et d'organisation du travail s'est pour ainsi dire envolée. La société Rover a été gérée comme une entreprise ordinaire et n'a pas saisi l'opportunité d'une coopération étendue entre les dirigeants et les salariés, qui aurait peut-être soutenu l'entreprise plus longtemps. 


\section{Bibliographie}

Armour J., Deakin, S. (2000), "The Rover case (2): bargaining in the shadow of TUPE", Industrial Law Journal, 29: 395-402.

Armour J., Deakin, S. (2001), "Norms in private insolvency procedures: the «London Approach» to the resolution of financial distress", Journal of Corporate Law Studies 1: 21-51.

Armour J., Deakin, S. (2003), "Insolvency and employment protection : the mixed effects of the Acquired Rights Directive", International Review of Law and Economics, $23: 1-23$

Brady C., Lorenz, A. (2020), End of the Road. BMW and Rover - A Brand Too Far, (London: Pearson).
Deakin S., Armour J. (2004), «Le sauvetage de Rover - une alternative au pouvoir de l'actionnaire?», L'homme et la société, 152/153, 79-86.

Deakin S., Morris G. (2005), Labour Law 4th, ed. (Oxford: Hart).

DTI (2005), Company Law Implementation of the European Directive on Takeover Bids: A Consultative Document, (Londres: Department of Trade and Industry).

Hart O., Moore J. (1997), "Default and Renegotiation: a Dynamic Model of Debt", NBER Working Paper 5907.

LEFrESNe F. (2005), «La faillite de MG Rover», Chronique internationale de l'IRES, $\mathrm{n}^{\circ}$ 94, mai, 5-11. 